\title{
First Aid Guidelines for Environmental Topics: Educational Approach and Adaptation
}

Lyle Karasiuk ${ }^{1}$ and Malini Nair ${ }^{2}$

${ }^{1}$ Canadian Red Cross, ${ }^{2}$ International Federation of Red Cross and Red Crescent Societies (IFRC)

Extreme environmental conditions and exposure to very high or low temperatures, particularly when this happens quickly, can lead to severe illness and injury. As the climate changes and temperature differences become more pronounced, these instances are likely to increase. First aid education which deals with environmental conditions is particularly important in circumstances where people are unprepared, more vulnerable due to age or have existing medical conditions. Remoteness or inaccessibility to medical care also increases vulnerability.

This paper reviews a selection of the environmental topics covered in the International First Aid, Resuscitation and Education Guidelines 2020 (IFRC, 2020) which seek to blend clinical first aid evidence with educational evidence, principles and expertise. Covering Hyperthermia, Dehydration, Hypothermia, Frostbite, Altitude Sickness, and Motion Sickness, we provide educational and adaptation interpretation in different contexts, stimulate debate and identify gaps. We also review how the 2020 Guidelines viewed these topics through the lens of the Chain of Survival Behaviors and identify the most pertinent stages in that chain to focus learning on these topics.

The 2020 Guidelines emphasize the need for content and educational adaptation according to the learner, which is central to these topics. First aid instructors and program designers need to frame action relevant to their audience and local needs. The teaching of environmental first aid topics should be contextualized to the local geography, legal requirements, culture and behaviors of the learners and their context.

For these topics, we conclude that facilitation tips for environmental conditions requiring first aid should focus on prevention and helping learners to recognize early warning signs of the different conditions so they can take preventative action before serious illness sets in.

\section{Introduction}

The International First Aid, Resuscitation and Educational Guidelines 2020 (IFRC, 2020 - hereafter referred to as the ' 2020 Guidelines') provide first aid educational methods that will support lay people to develop skills, knowledge and behavioral change to be able to respond to such emergencies. They are designed to be a foundation resource for first aid education providers. National Societies and others within the Red Cross and Red Crescent Movement (hereafter referred to as 'the Movement') are expected to adapt their education and practice set out in the 2020 Guidelines in accordance with the local
Karasiuk \& Nair 2021.

Creative Commons

AttributionNonCommercial 4.0 International License

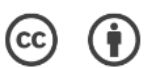


environmental, legal, cultural, linguistic and technological context. The 2020 Guidelines are also open and free to be used by any other first aid education providers.

The environmental topics reviewed in this paper include Hyperthermia, Dehydration, Hypothermia, Frostbite, Altitude Sickness, and Motion Sickness. The purpose of this review paper is to provide educational and adaptation interpretation in different contexts, stimulate debates, and identify any gaps. This paper also reviews how the guidelines incorporated available clinical and educational evidence within the domains of the Chain of Survival Behaviors (IFRC 2016, see Figure 1) by first aid topic. It then discusses how the educational considerations might be adapted to different learner audiences and their needs.

Extreme environmental conditions or change in weather patterns (extreme cold and heat) have been associated with change in climate, mortality and morbidity. It is expected that the intensity and frequency of heat waves and extreme cold will continue to increase in the future because of changing weather patterns (Parry et. al, 2007). Public health problems and emergencies become apparent through exposure to extreme natural heat resulting in heat exhaustion, heat stroke, heat cramps and heat related deaths (Berko et. al, 2014). Extreme exposure to natural heat and cold can be also detrimental to and exacerbate pre-existing medical conditions such as respiratory and heart diseases. Infants, elderly and people with medical conditions or pre-existing conditions are most vulnerable. The elderly population is at a significantly higher risk of hypothermia and hyperthermia (Kare \& Shneiderman, 2001). The human body works well at a core temperature of about 37 degrees centigrade, and the core area that includes vital organs may cease to function and become life threatening if its temperature moves too far above or below this (NZRC, 2017).

In situations where a person is afflicted with hypothermia or hyperthermia, immediate care is needed meaning one must act early and quickly as

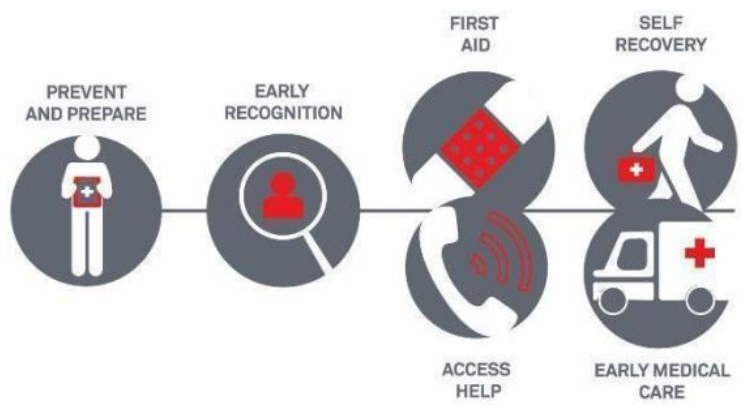

Figure 1: The Chain of Survival Behaviors

these can be life threatening. A person exposed to extreme natural heat and cold can become unconscious and stop breathing. Remoteness, inaccessibility and lack of transportation can be a challenge: knowing simple steps can help save lives. First aid services and local first aid providers can respond to potential emergencies as such when accessibility and remoteness is an issue.

The International Federation of the Red Cross and Red Crescent (IFRC) updated their 2016 Guidelines in February 2021 to reflect the evidence specific to first aid clinical actions and uniquely paired evidence regarding first aid education. This approach was designed so that users of the guidelines are easily able to apply and adapt to local and learner contexts, contextualize the availability of resources and medical care, and local legal requirements and strategies.

The development of each new guideline and the review of the 2016 version included a new focus on the domains of the Chain of Survival Behaviors (IFRC 2016) as well as a fresh look through an educational lens across different contexts and the provision of education considerations for each topic. This shift in emphasis occurred in response to calls from curriculum developers and first aid educators from across the Movement (including Red Cross National Societies and the International Committee of the Red Cross - ICRC). The process to include these elements was rigorous and consistent and is explained in full in the 2020 Guidelines. Topics for inclusion were identified in 2018 using surveys and expert opinion of actors within the Movement. Individuals from 43 countries with clinical and/or educational expertise participated in teams to 
develop search criteria from existing published literature and grey literature for each research question.

Following a clinical review of the evidence, each topic was then considered independently by at least two educational reviewers (including one from a lower resource setting and one from a higher resource setting) who reviewed existing literature to inform the educational approach to a first aid intervention for that topic. Experience and expertise of those undertaking the evidence reviews were also drawn upon. The contributions synthesized the insight available and contributed educational aspects to the Chain of Survival Behaviors for the topics. The guideline includes education considerations to support the application of the topic in an educational setting. Conflicts were resolved by an independent Guidelines Steering Committee (GSC) member. Worksheets that described the outcomes and relevant summary were completed, presented for peer review.

Each condition is introduced with a key action to be emphasized to learners, a brief explanation of the condition, how common it is and the main population likely to be at risk or affected. The 2020 Guidelines that follow are rated by the level of scientific evidence that exists for them. Good practice points are provided where evidence was missing but expert opinion was available based on experience and non-systematic review sources of evidence.

The Chain of Survival Behaviors is used by the 2020 Guidelines authors as a tool to emphasize how first aid education does not start and end with a first aid action. The domains of Prevent and Prepare; Early Recognition; First Aid and/ or Access help; and Early Medical Care/ Self-recovery, are fundamental considerations for education depending on the context, availability of resources and learner needs. Each clinical topic covered by the 2020 Guidelines includes insight based on evidence and expert opinion on how best to frame an educational intervention using these domains and identifying where educators might best position that learning.
Thus, the major audience for the 2020 Guidelines are first aid program designers, program managers, education and scientific committees and trainers.

\section{Educational approach}

For Environmental topics, the consistent advice is to change the environment that the person is in which is making them ill or injured: warming the person who is cold, cooling the person who is warm, bringing the person who has altitude sickness to a lower level, and bringing the person with motion sickness to stillness. While each of these topics has different ways to approach the context and the 'treatment' required, the removal of the person from the situation is deemed the key action in all cases.

\section{Hyperthermia}

The first aid guideline is to rapidly cool the person using immersion in cold water $\left(1-26^{\circ} \mathrm{C}\right)$ from the neck down or by another active cooling technique (Douma 2020; CEBaP, 2020). Education facilitators are advised to focus on prevention and preparation, such as wearing appropriate clothing for the climate and limiting physical activity during the hottest part of the day. Recognition of the signs and symptoms of hyperthermia are presented as 'mild to moderate hyperthermia (heat exhaustion)' and 'severe hyperthermia (heatstroke). Facilitators are also encouraged to focus on early recognition of hyperthermia so that learners can intervene before the condition becomes severe. Contexts are important for this topic from both a legal and cultural perspective (such as around taking off the person's clothes to cool them), and a resource perspective what can be done when cold water is not available, or water is insufficient for the demand.

\section{Dehydration}

Dehydration is a shortage of water in the body. The 2020 Guidelines suggest rehydrating the person with water in mild cases and or commercially prepared oral rehydration salts (ORS) or a pre-prepared salt package that complies with the World Health 
Organization's advice for significant dehydration and or extreme vomiting and diarrhea. The first aid guideline is to remove any excessive clothing if the person is under heat stress or a hot environment and give them fluid. Recognition of signs and symptoms of dehydration are presented as mild to severe. When the body is dehydrated, it can experience cramps, changes in mental capacity or even shock (which can be life-threatening if left untreated), especially in small children or older adults. Significant loss of water causes the person to lose electrolytes, which are essential for the body to function normally.

Education facilitators should focus on early recognition and highlight different types of environmental conditions or situations where dehydration can take place such as heatwaves, people doing vigorous physical activities and may not have adequate access to fluids or having severe diarrhea and vomiting.

Education facilitators should define 'dehydration' to the learner in the context of their region and the likely ways people could become dehydrated. As part of the Chain of Survival Behaviors, facilitators should focus on prevention and preparation to ensure keeping the body hydrated. Whatever the context, the learner needs to understand how to motivate people with mild dehydration to drink enough fluids (e.g., water or diluted apple juice in children older than 6 months). The context in which dehydration is likely to occur varies greatly. Educators should adapt to their audience and frame the education they provide accordingly. For example, learners who might work outdoors on a construction site must be advised to drink plenty of fluids and take breaks, albeit recognizing that they might not have this possibility as the work must go on. Safe work practices such as frequent breaks, shelter or reduced/different hours, might be options to consider. In contrast, someone who might need to exercise, or undertake simple chores outdoors could choose the cooler time of the day or opt not to go out. Staying cool and drinking fluids is essential and educators are advised to discuss how this can best be achieved in the situation that learners might face.
The use of breast milk for babies as a safe form of hydration should be advocated as this does not rely on potable (clean) water. If milk formula is the only available source of milk, emphasize the need for the water to be potable (clean) to avoid infections that cause vomiting and diarrhea. In the context of the learners, educators need to emphasize to new mothers that diarrhea and vomiting in infants and children can have disastrous effects if left to "correct itself" if it continues for more than 6-8 hours. Breastfeeding for babies should be continued.

In 2015, the International Liaison Committee on Resuscitation (ILCOR) completed a systematic review on the use of carbohydrate-electrolyte solutions for people with exertion-related dehydration (Singletary, 2015 and Zideman, 2015). The review included 12 studies that showed that drinking $5 \%$ to $8 \%$ (8 studies) or $3 \%$ to $4 \%$ (3 studies) carbohydrate-electrolyte solutions facilitates rehydration after exercise-induced dehydration. Evidence also showed that participants generally tolerated ingesting these solutions.

We cannot overemphasize the need to keep hydrated and take measures to stay cool in hot weather by seeking respite from the sun either indoors or in appropriate shade. With increased activity there needs to be an equal increase in fluid consumption to that which is lost.

\section{Hypothermia}

Hypothermia is a condition in which the body's core temperature drops below $35^{\circ} \mathrm{C}\left(95^{\circ} \mathrm{F}\right)$ and cannot function properly: the blood circulation reduces significantly, especially in the small vessels in the skin. It can occur when a person is exposed to extreme cold, such as in mountainous regions in the winter. Other factors that intensify the risk of hypothermia are living in homes that lack heating, the use of alcohol or drugs or pre-existing mental health conditions. Some conditions reduce people's ability to recognize when they have lost a significant amount of heat. Hypothermia occurs in many parts of the globe, particularly northern regions. As well as being more predictable in areas with extremely low temperatures, it can also occur when a person is not 
adequately dressed and unexpectedly becomes stranded and is forced to walk for help, or in those working outdoors for prolonged periods in lower temperatures. Other instances are seen in those who lose heat/power due to natural causes or poor living conditions in a cold climate.

Educators need to frame the context of staying warm, getting warm and keeping warm with the area of living, actions of living and societal norms. The basics of food, shelter and clothing are essential to care in hypothermia. Just as for hyperthermia where hot dry skin is a sign to be taken seriously, no shivering when a person has hypothermia indicates an extreme emergency. Those with severe hypothermia may also suffer significant frostbite in unprotected limbs such as fingers and toes. Educators need to emphasize the importance of preventative action such as wearing appropriate clothing, staying indoors when outside temperature is cold, having proper mechanical heating for residences, and having the ability to build a fire to generate heat if stranded or lost in the wilderness. Basic human needs of food and shelter are essential to survival in the cold.

\section{Frostbite}

When it is cold (at or below $0^{\circ} \mathrm{C}$ ) or there are strong winds, the human body tries to preserve its core body temperature by narrowing the blood vessels close to the skin. In extreme cases, this can reduce the blood flow in some areas of the body to dangerously low levels resulting in damage to the skin and other tissues. The fingers and toes are the most vulnerable. Frostbite can lead to permanent damage, but this can be avoided if it is recognized and treated quickly. A person with frostbite may also be experiencing Hypothermia.

Educators should explore safe behaviors and preparedness in very cold climates relevant to learners, for example, being stuck somewhere remote with a broken-down vehicle in winter, working in wilderness areas, or doing winter sports such as skiing. Where appropriate, educators should target those more vulnerable such as elderly and homeless people. As frostbite is relatively uncommon, resource materials such as images could usefully be used to illustrate the condition. Scenario learning can also help learners to identify how they would get medical help in an emergency in the extreme cold.

Guidelines produced by the Wilderness Medical Society on the prevention and management of frostbite provide recommendations based on case series and animal studies (McIntosh et al., 2019). The evidence from Wilderness Medical Society has been incorporated in the 2020 Guidelines as well. The recommendations include:

(1) Field rewarming by warm water bath immersion can and should be performed if the proper resources are available and medical care is more than two hours distant. Other heat sources (e.g., fire, space heater, oven, heated rocks) should be avoided because of the risk of thermal burn injury;

(2) Rapid rewarming by water bath has been found to result in better outcomes than slow rewarming;

(3) Field rewarming should only be undertaken if the frozen part can be kept thawed and warm until the person arrives at medical care. Water should be heated. Circulation of water around the frozen tissue will help maintain the correct temperature.

There is limited evidence but tropical aloe vera may be beneficial when applied to the frostbite. (Heggars et al., 1987; McIntosh, 2019; McCauley, 1983.)

\section{Motion Sickness}

Motion sickness is caused when the brain's estimate of motion is different from what the person is experiencing. The person's eyes, balance center of the inner ear and general perception of their body's position and movement conflict with the messages the brain receives. Motion sickness often presents with nausea and vomiting which need to be controlled or resolved.

Sensitivity to motion sickness varies in individuals, but most people will experience it if the cause is strong enough. Pregnant women, children older than two years and those suffering with migraines are most susceptible. First aid care is often ineffective, and educators should emphasize prevention instead, 
such as not reading when a vehicle is in motion and not riding backwards on a train. Although there is a lack of scientific evidence available, some natural remedies might also be helpful. While the 2020 Guidelines do not support "folk remedies" without an evidence base, traditional medicine cannot be overlooked. Rather, we need to identify this as a gap and, where possible, incorporate traditional practices in future guidelines.

\section{Altitude sickness}

Altitude sickness occurs when people at a high altitude do not have enough oxygen in their blood because the air pressure is too low. As altitude increases, the air becomes thinner, and less oxygen is inhaled with each breath. The term altitude sickness includes acute mountain sickness (AMS), high altitude pulmonary oedema (HAPE) which affects the lungs and breathing, and high-altitude cerebral oedema (HAKE) which affects the brain, behavior and alertness. Individuals most affected by altitude sicknesses are those who travel to a high altitude quickly (e.g., tourists), especially if they have preexisting medical conditions. However, trained mountaineers can also develop symptoms when they reach very high altitudes, such as in the Himalaya region.

Based on a small randomized controlled trial with 34 healthy mountaineers, gradual ascent to higher altitudes seemed a more effective method of prevention than a fast ascent (Bloch, 2009). Educators need to emphasize that both ascent and descent need to be slow and gradual to allow the body to adapt to the change. In much the same way that the rapid ascent of a diver can cause serious complications known as the bends, rapid ascent can equally be a challenge. Proper precautions and equipment must be used for those climbers. Advice should also be given to tourists who take a cable car to a significantly higher observation platform to slowly engage in activity allowing their bodies to adjust to the new altitude. Excessive exertion should be avoided and the opportunity for regular breaks should be encouraged.

\section{Adaptations}

To better adapt the education approaches, educators and learners must understand what the situation and context is, the surrounding environment and what signs and symptoms are visible. The educators should also support learners to critically think on how to use the available local resources to prevent such a situation and minimize/prevent further harm. The "why" context relates to why first aiders endeavor to avoid conditions from becoming worse, reflecting the aims of first aid: to prevent further injuries, preserve and promote recovery.

To apply the first aid guidelines in the context of environmental consideration, one needs to understand the climate, culture and situations which a first aider will face. Knowing that on the Prairies of Canada wind chills can be $-50^{\circ} \mathrm{C}$ might be irrelevant to someone in the southern hemisphere. Similarly, learners facing the hot sun of the desert climate where temperatures can be the opposite extreme need strategies that are relevant and effective in such a context. For example, people living in tropical climates, such as in the Pacific region or Sub-Saharan Africa, can experience high cases of dehydration due to its weather patterns and climate. These conditions can cause a significant loss of water in the forms of sweat and other body fluids. Dehydration can also occur in cold settings when people wear too many layers or overexert themselves (IFRC, 2020). Depending on the availability and access to potable (clean) water, educators may need to include information on water sterilization methods (e.g., boiling or chlorination) and the risks of drinking contaminated water. Information on the importance of drinking fluids which rehydrate the body, such as water and juice should be included considering their country context. Educators must understand where the learners are from, what parts of the climate they can relate to and how best can they resolve the need to safely care for someone. They need to also view the context of the culture. Where access to emergency services via a dedicated system like 9-1-1 might send resources to my door, others might be faced with a long walk to a nursing station just to 
summon the help, let alone access definitive care, and medications might not be readily available. Each learner should be supported to think during the first aid training about how likely they are to encounter this type of an emergency and what they can realistically do to act effectively, particularly where access to clean water, a safe environment, and safe medical care is not easily nor readily accessible.

For the environmental conditions described in this review, home remedies, sometimes referred to as folk remedies, can be beneficial, as for centuries the caring traditions have been passed down through the family. How do we incorporate these remedies into the context of caring for the sick and injured? Their impact may often be overlooked on the success of a person's care. For example, for dehydration, education facilitators are encouraged to focus on home-made oral rehydration solutions (ORS) or other locally available liquids such as coconut water or tea. While some cultures also dismiss these practices as pure folklore and having no relevance, it remains important to consider when and how best to incorporate or balance the traditional methods with the 'modern' medicine.

We need to prepare our learners for the world/environment for which they will encounter. How educators adapt skills and knowledge will also reflect the learner outcomes set by educators or facilitators. The learner outcomes are changes that occur in the learner's knowledge, skills and attitudes or beliefs because of their learning experience (Boase, Gordon \& Oliver, 2021). These authors further suggest that investing into evaluating learner outcomes, facilitators and educators of an organization can really start to improve the effectiveness of first aid education. The aim is to create individuals and communities who are confident, able, and willing to respond to first aid emergencies and are more likely to respond in a first aid emergency. To be more inclusive, instructions to learners should focus on what needs to be achieved (i.e., the outcome) rather than the exact way something must be done (IFRC, 2020).
Climate change is very real and affects many people around the world. There is the real likelihood that environmental emergencies become more severe, common or predominant in places never previously encountered. Higher temperatures for longer periods are just one example with increased drought already occurring in some places. The community needs to examine ways to research this further but also look at ways to educate learners in areas where they may have never experienced these environmental conditions.

Educators need to give people the experiences of learning through the lens of their own country or community context. The phrase 'to have walked a mile in my shoes' to understand what it's like to be in that situation is a relevant and realistic experience and can help educators to come across as authentic.

\section{Discussion}

Inclusiveness and education are the major strengths of the 2020 Guidelines. How educators are supported with practical steps to provide first aid education and care is vital. A driving principle of effective first aid education is to provide variety, simplicity, clarity, and to be outcome-led, focusing on content that is relevant to learners and their contexts.

The 2020 Guidelines are framed around the Chain of Survival Behaviors (CoSB), and for the topics reviewed in this paper there tends to be limited evidence for each of the interventions provided. In most cases interventions have not been tested against each other adequately or across different population groups. Linked to this, and also lacking, is adequate evidence relating to how best to address social issues through first aid education. For example, the prevention of dehydration might be possible through particular behavioral changes suggested to learners, but fundamental issues of poor nutrition, unclean or unavailable water, and lack of medical care might be intrinsically linked to the likelihood of dehydration and associated illnesses in some communities and first aid will not solve these problems. Educators are 
well advised in the 2020 Guidelines to work with learners to identify risks and solutions where possible.

A further challenge for educators on these topics occurs in the 'recognition' domain of the CoSB. Environmental conditions covered in this review generally develop over time (although this is highly variable) and might be hard to recognize in the early stages. Signs might also easily be missed or mistaken for something else, such as drunkenness. Again, context and behaviors need to be incorporated into learning to minimize the likelihood of mistaken diagnosis by the first aid responder.

The third domain of the CoSB is the provision of help or access to help. The environmental conditions described in this review are particularly poignant ones for more fragile contexts - areas which are remote or hard to reach, where calls for help are harder to respond to and removal from the situation might be logistically impossible. Educators preparing to instruct learners facing such situations, might be advised to also consult the 'Contexts' section of the 2020 Guidelines which gives ideas for scenario-based activities in remote, disaster and conflict contexts, amongst others. Such activities can be particularly helpful to learners who are not used to a particular context or are unfamiliar with response mechanisms and options.

So, while we often look at the science to point us to the highest standards of treatment and care, those developing first aid education need to consider how to adjust advice to take account of social determinants of health and local conditions. Framing education in a way that allows learners to feel confident that they are doing the best they can in a challenging situation outweighs the importance of teaching them the 'correct' way to act when the resources to do so are not available. For future guidelines, it might be interesting to consider culture and social determinants of health more deeply and understand how first aid can best be used to help people most effectively.

\section{Conclusion}

The 2020 Guidelines allow organizations to adapt to different kinds of needs and contexts. The outcomebased descriptions of the first aid steps are inclusive of people with diverse physical needs and abilities. The way actions are described for the first steps are based on what needs to be achieved rather than the way something needs to be done and this makes them valuable for educators working with different learner needs and levels of resources. Program designers are encouraged to consider cultural and legal implications of first aid guidelines, and tailor their approach appropriately.

For further consideration on encouragement to adapt programs to the needs and abilities of learners, conscious efforts should be made to improve inclusivity related to gender, age and resource integration. The 2020 Guidelines encourage program designers to adapt first aid skills to the abilities of learners, with guidance that adaptations need to "allow the first aid provider to perform the principles of the method safely and effectively, be safe for the ill or injured person and be quick to start".

Facilitation tips for environmental conditions requiring first aid should focus on prevention and help learners to recognize early warning signs of the different conditions so they can take preventative action before serious illness sets in.

There is no 'one size fits all' approach presented in the 2020 Guidelines but rather they are framed as a set of 'principles' or relevant 'dos and don'ts' that must include a local, regional, or cultural flair. We must learn to take lessons learned from others' best practices and create new learnings applicable to our own region.

There is a big, bold, diverse world that challenges us to meet needs in our own part of the globe. Adapting and overcoming those challenges is a big part of first aid education.

\section{Acknowledgements}

We wish to thank the reviewers, writers and editors that were involved in the development of the 2020 Guidelines. 


\section{Conflict of Interests}

The authors were content contributors to the International First Aid, Resuscitation, and Education Guidelines 2020. Thus, there are no conflicts of interest.

\section{Corresponding Author}

Lyle Karasiuk, National Volunteer Chair, Canadian Council for First Aid Education, Canadian Red Cross. lyle.karasiuk@,redcross.ca

\section{References}

Berko, J, Ingram, D. D., Saha, S., Parker, J. D., (2014). Deaths attributed to heat, cold, and other weather events in the United States, 2006-2010. National health statistics reports; no 76. Hyattsville, MD: National Center for Health Statistics.

Boase, C., Gordon, E. and Oliver E. (2021). A toolkit for National Societies to support improvement of first aid education, Global First Aid Reference Centre in collaboration with the British Red Cross, https://www.globalfirstaidcentre.org/wp-content/uploads/2020/12/EN Outcomes-MeasurementToolkit-1.pdf

Centre for Evidence-Based Practice, Belgian Red Cross-Flanders. (2020). Evidence summary Dehydration - Home-made ORS. Available from https://www.cebap.org/knowledge-dissemination/first-aidevidence-summaries/

Centre for Evidence-Based Practice, Belgian Red Cross-Flanders. (2016). Evidence summaries to support. First Aid Guidelines. Dehydration - ORS. Available from: https://www.cebap.org/knowledgedissemination/first-aid-evidence-summaries/

Douma, M. J., Aves, T., Allan, K. S., Bendall, J. C., Berry, D. C., Chang, W. T., ... Lin, S. (2020). First aid cooling techniques for heat stroke and exertional hyperthermia: A systematic review and meta-analysis. Resuscitation, 148, 173-190.

Heggers, J. P., Robson, M. C., Manavalen, K., Weingarten, M.D., Carethers, J.M., Boertman, J.A., ... \& Sachs, R.J. (1987). Experimental and clinical observations on frostbite. Annals of emergency medicine, 16(9), 1056-1062.

Kare, J. A., Shneiderman, A. (2001). Hyperthermia and Hypothermia in the Older Population, Topics in Emergency Medicine: September 2001 - Volume 23 - Issue 3 - p 39-52

McCauley, R. L., Hing, D. N., Robson, M. C., \& Heggers, J. P. (1983). Frostbite injuries: a rational approach based on the pathophysiology. The Journal of trauma, 23(2), 143-147.

McIntosh, S. E., Freer, L., Grissom, C. K., Auerbach, P. S., Rodway, G. W., Cochran, A., ... \& Pandey, P. (2019). Wilderness Medical Society Clinical Practice Guidelines for the Prevention and Treatment of Frostbite: 2019 Update. Wilderness \& environmental medicine, 30(4), S19-S32. https://www.wemjournal.org/article/S1080-6032(19)30097-3/fulltext

New Zealand Red Cross. (2017), Essential First Aid Manual, ISBN 978-0-908998-26-5,. https://media.redcross.org.nz/media/documents/NZRC Essential First Aid Manual TR35-010$\underline{012021 \text { v5.pdf }}$ 
Review

Parry M. L., Canziani O.F., Palutikof J. P., van der Linden P. J., Hanson C. E. (editors), (2007). Contribution of Working Group II to the Fourth Assessment Report of the Intergovernmental Panel on Climate Change, 2007. Cambridge, UK and New York, NY: Cambridge University Press. 